\title{
An epistemic interpretation of quantum probability via contextuality
}

\author{
Claudio Garola \\ Department of Mathematics and Physics, University of Salento \\ Via Arnesano, 73100 Lecce, Italy \\ e-mail: garola@le.infn.it
}

June 26, 2018

\begin{abstract}
According to a standard view, quantum mechanics (QM) is a contextual theory and quantum probability does not satisfy Kolmogorov's axioms. We show, by considering the macroscopic contexts associated with measurement procedures and the microscopic contexts ( $\mu$-contexts) underlying them, that one can interpret quantum probability as epistemic, despite its non-Kolmogorovian structure. To attain this result we introduce a predicate language $L(x)$, a classical probability measure on it and a family of classical probability measures on sets of $\mu$-contexts, each element of the family corresponding to a (macroscopic) measurement procedure. By using only Kolmogorovian probability measures we can thus define mean conditional probabilities on the set of properties of any quantum system that admit an epistemic interpretation but are not bound to satisfy Kolmogorov's axioms. The generalized probability measures associated with states in QM can then be seen as special cases of these mean probabilities, which explains how they can be non-classical and provides them with an epistemic interpretation. Moreover, the distinction between compatible and incompatible properties is explained in a natural way, and purely theoretical classical conditional probabilities coexist with empirically testable quantum conditional probabilities.
\end{abstract}

Key words: quantum probability, contextuality, entanglement, quantum measurements.

\section{Introduction}

There are some typical features of quantum mechanics (QM) that are well established and accepted in the current literature but still raise interpretative problems. We are especially interested here in the following topics.

(i) Non-kolmogorovian character of quantum probability, implied by the nondistributivity of the lattice of (physical) properties, which is the basic structure of standard quantum logic (QL). 
(ii) The doctrine that, whenever a physical system in a given state is considered, a quantum observable generally has not a prefixed value but only a set of potential values, and that a measurement actualizes one of these values, yielding an outcome that depends on the specific measurement procedure that is adopted (contextuality).

There is a huge literature on these topics, which goes back to the early days of QM. We limit ourselves here to recall that the QL issue was started by a famous paper by Birkhoff and von Neumann (1935), while the contextuality at a distance (or nonlocality) and, more generally, the contextuality of QM were accepted by most physicists as "mathematically proven" after the publication of Bell's $(1964,1966)$ and Kochen-Specker's (1967) theorems, later supported by numerous different proofs of the same or similar theorems (among which the famous proof of nonlocality provided in 1990 by Greenberger, Horne, Shimony and Zeilinger, which does not resort to inequalities).

Non-classical probability and contextuality can be linked, and inquiring their links leads to important achievements. This issue has been already studied by the "Vāxjö school", and in particular by Khrennikov (2009). We propose in this paper a new perspective, according to which quantum probability and its nonclassical features can be interpreted as derived notions in a classical probabilistic framework by taking into account microscopic and macroscopic contexts.

To the best of our knowledge, our proposal is innovative. Let us therefore summarize the essentials of it.

First of all, we introduce some epistemological and physical remarks on QM in Section 2 by referring to a conception of QM according to which QM deals with individual examples of physical systems (briefly, individual objects) and their properties (see, e.g., Busch et al., 1996). Bearing in mind these remarks, we work out in Section 3 a predicate language $L(x)$ whose predicates either denote states or pairs made up of a property $E$ and a (generally unknown) microscopic context ( $\mu$-context) $C$. Hence the elementary sentences of $L(x)$ assert that the individual object $x$ is in a given state or that $x$ has a given property in a given $\mu$-context, but not that $x$ has a given property without reference to contexts, as in the standard language of QM. Then we introduce a classical notion of probability on the set of all sentences of $L(x)$ in Section 4 and a family of classical probability measures on sets of $\mu$-contexts in Section 5 , each element of the family corresponding to a measurement procedure that determines a macroscopic measurement context. We can thus define a notion of compatibility on the set $\mathcal{E}$ of all properties, hence a notion of testability on the set of all sentences of $L(x)$, and use the foregoing probabilities conjointly to define the notion of mean conditional probability on the subset of all testable sentences of $L(x)$ and the related notion of mean probability measurement. The former admits an interpretation that is epistemic (in a broad sense, i.e., relating to our degree of knowledge/lack of knowledge), even if it is not bound to satisfy Kolmogorov's axioms because it is obtained by averaging over classical probability measures.

Based on the definitions and results expounded above, we focus in Section 6 on the set $\mathcal{E}$ of all properties, on which mean conditional probabilities induce a 
preorder relation $\prec$. We show that, if suitable structural conditions are satisfied, a family of mean conditional probabilities can be introduced, parametrized by the set $\mathcal{S}$ of all states, each element of which is a generalized probability measure on $(\mathcal{E}, \prec)$. Moreover these measures allow the definition of a new kind of conditioning referring to a sequence of measurement procedures that is conceptually different from classical conditioning.

The formal scheme described above characterizes a broad class $\mathcal{T}$ of theories. Then we assume in Section 7 that QM belongs to $\mathcal{T}$, so that states and properties can be interpreted as quantum states and quantum properties, respectively, and the quantum probability measures associated with states can be considered as the specific form that the generalized probability measures defined on $\mathcal{E}$ take in QM. Hence we attain the following results.

(i) The nonclassical character of quantum probability can be explained in classical terms by taking into account $\mu$-contexts. It follows in particular that quantum probability can be given an epistemic rather than an ontic interpretation in our approach 1

(ii) The quantum relation of compatibility on the set of properties can be considered as the specific form that the relation of compatibility introduced in the general framework takes in QM.

(iii) The conditional probability usually introduced in QM can be considered as the specific form that the new kind of conditioning introduced in the general framework takes in QM.

We conclude our treatment by observing in Section 8 that the general notions of mean conditional probability and mean probability measurement are conceptually close to the notions of universal average and universal measurement, respectively, introduced by Aerts and Sassoli de Bianchi (2014, 2017). Hence our approach provides a description of measurements of probabilities that is similar to the proposal of these authors, which they maintain to supply a possible solution of the hoary quantum measurement problem. We however do not make such a claim in the case of our approach, because we supply our definition of mean probability measurement resting on the standard notion of measurement in QM, without entering the problematic aspects of this notion (as state reduction and nonlocality) which arise when QM is assumed to refer to individual objects and their properties. Nevertheless the results expounded above are sufficient in our opinion to justify our proposal.

To close this section, let us point out an essential difference between our approach and Khrennikov's. This author considers contexts 'as a generalization of a widely used notion of preparation procedure' (2009b). As we have seen, we introduce instead measurement procedures determining macroscopic measurement contexts, each of which is associated with a set of microscopic contexts. The latter play an essential role in our framework, as they allow us to obtain the results resumed above, and do not occur in Khrennikov's approach.

\footnotetext{
${ }^{1}$ We stress that our general framework does not constitutes a hidden variables theory for QM in a standard sense. Indeed, $\mu$-contexts are associated (generally many-to-one) with measurement procedures, not with properties or states of the measured entity. Our perspective complies instead with Aerts' (1986) hidden measurements approach.
} 


\section{Some remarks on $\mathrm{QM}$}

As other advanced scientific theories, QM is expressed by means of a fragment of the natural language enriched with technical terms (the language of $Q M$ ) and is characterized by a pair $(F, I)$, with $F$ a logical and mathematical formalism and $I$ an empirical interpretation which establishes connections between $F$ and an empirical domain. This interpretation generally is indirect, in the sense that there are theoretical entities that are connected with the empirical domain only via derived theoretical entities, and incomplete, in the sense that only limited ranges of values of the theoretical entities are actually interpreted 2

To attain the results summarized in Section 1, we need to formalize an elementary sublanguage of the language of QM. Let us therefore preliminarily discuss some features of this language and some intuitive ideas on its interpretation, referring to a conception of QM according to which QM deals with individual objects and their properties, as we have anticipated in Section 1. For the sake of simplicity we avoid distinguishing everywhere in the following the theoretical entities from the empirical entities that correspond to them via $I$.

First of all we recall that in most presentations of QM the notions of physical system, or entity, (physical) property and (physical) state are considered as basic. Moreover, according to some known approaches to the foundations of QM (see, e.g., Beltrametti and Cassinelli, 1981; Ludwig, 1983) states are considered as classes of probabilistically equivalent preparation procedures, or preparing devices, and properties as classes of probabilistically equivalent dichotomic (yesno) registering devices.

A preparation procedure $\pi$ in the class $S$, when activated, produces an individual object $x$ (which can be identified with the act of activation itself if one wants to avoid ontological commitments). Hence, after the act of activation, a sentence that states that $x$ is in the state $S$ is true and a sentence that states that $x$ is in a state $T \neq S$ is false.

Given an individual object $x$ in the state $S$, however, activating a registering device $r$ in the class $E$ does not test whether the property $E$ is possessed or not by $x$ independently of the simultaneous activation of other devices. Indeed it follows from some known proofs of the Bell and Kochen-Specker theorems mentioned in Section 1 (see, e.g., Greenberger et al., 1990; Mermin 1993) that, if the laws of QM have to be preserved in every conceivable physical situation,

\footnotetext{
${ }^{2}$ More generally, according to the standard epistemological conception, or received view (see, e.g., Braithwaite, 1953; Hempel, 1965; Carnap, 1966), a fully-developed physical theory $T$, as QM, is expressed by means of a metalanguage in which a theoretical language $L_{T}$, an observational language $L_{O}$ and correspondence rules $R_{C}$ connecting $L_{T}$ and $L_{O}$ can be distinguished. The theoretical apparatus of $T$, expressed by means of $L_{T}$, includes a mathematical structure and, usually, an intended interpretation that is a direct and complete physical model of the mathematical structure. The observational language $L_{O}$ describes instead an empirical domain, hence it has a semantic interpretation, so that the correspondence rules $R_{C}$ provide an empirical interpretation of the mathematical structure that is indirect and incomplete in the sense specified above.

The received view has been criticized by several authors (see, e.g. Kuhn, 1962; Feyerabend, 1975 ) and is nowadays maintained to be outdated by some scholars. However, we retain here some of its basic ideas that we consider epistemologically relevant.
} 
the outcome that is obtained depends on the set of the registering devices that are activated together with $r$, i.e., on the macroscopic context $\boldsymbol{C}_{M}$ determined by the whole quantum measurement $M$ that is performed (of course, these registering devices must be compatible, i.e., they must belong to different but compatible properties). Hence, one must admit that, generally, a truth value can be assigned to a sentence which states that $x$ possesses the property $E$ only if also a macroscopic context is specified (contextuality of QM) 3

We observe now that, generally, the macroscopic context $\boldsymbol{C}_{M}$ determined by $M$ may be produced by many different microscopic physical situations that cannot be distinguished at a macroscopic level (though they can be described, in principle, by $\mathrm{QM}$ itself). Hence we can associate $\boldsymbol{C}_{M}$ with a set $\mathfrak{C}_{M}$ of microscopic contexts ( $\mu$-contexts; of course, $\mathfrak{C}_{M}$ could reduce to a singleton in special cases). It is then natural to think that the truth value of a sentence asserting that $x$ possesses the property $E$ generally depends on the $\mu$-context that is realized when $M$ is performed. But we cannot know this $\mu$-context, hence only a probability of it, which expresses our degree of ignorance, can be given (we naively argue here as though the set $\mathfrak{C}_{M}$ were discrete, to avoid technical complications).

Summing up, our analysis leads us to conclude that a truth value can be assumed to exist consistently with QM only in the case of a sentence asserting that an individual object $x$ possesses a property $E$ in a given $\mu$-context $C$, not in the case of a sentence simply asserting that $x$ possesses a property $E$. Moreover, in general this value cannot be deduced from the laws of QM, which are probabilistic laws that make no explicit reference to contexts.

The conclusions above have an important consequence. Every quantum prediction concerns probabilities, hence testing it requires evaluating frequencies of outcomes. In our present perspective, a typical test of this kind consists in preparing a broad set of individual objects in a given state $S$ and then performing on each of them the same quantum measurement $M$, which requires activating one or more (compatible) registering devices simultaneously. The macroscopic context $\boldsymbol{C}_{M}$ then is the same for every individual object, but the $\mu$-context $C \in \mathfrak{C}_{M}$ generally changes in an unpredictable way. Thus we meet

\footnotetext{
${ }^{3}$ We have emphasized in some previous papers (see, e.g., Garola, 1999; Garola and Pykacz, 2004; Garola and Sozzo, 2010; Garola and Persano, 2014) that the epistemological clause "the laws of QM have to be preserved in every conceivable physical situation" is essential in the proofs of Bell's and Kochen-Specker's theorems. Nevertheless, this clause generally is not explicitly noticed or stated, possibly because it seems to be unquestionably justified by the outstanding success of QM. Yet it must be observed that all the proofs mentioned above proceed ab absurdo, hypothesizing physical situations in which noncompatible physical properties are assumed to be simultaneously possessed by an individual object. In such situations the quantum laws that are applied cannot be simultaneously tested, hence the assumption that they hold anyway seems more consistent with a classical than with a quantum view. One can therefore try to give up the aforesaid clause, but then the proofs of Bell's an Kochen-Specker's theorems cannot be completed. This conclusion opens the way to the attempt at recovering noncontextual interpretations of QM (Garola, Sozzo and Wu, 2016). The arguments in this paper, however, apply to every theory in which contexts can be defined, irrespective of whether the results of measurements are context-depending (locally, or also at a distance) or not.
} 
two distinct sources of randomness. The first is the state $S$ (be it a pure state or a mixture) that may not determine univocally the properties of an individual object in QM, even if the $\mu$-context $C$ is given. The second is the unpredictable change of the $\mu$-context that occurs when performing $M$ on different individual objects. We are therefore led to think that quantum probability takes implicitly into account both sources. We will see in the following sections that this idea can explain the non-kolmogorovian character of quantum probability, together with the rather surprising fact that the values of quantum probability neither depend on $\mu$-contexts nor on macroscopic contexts (see, e.g., Mermin, 1993).

\section{The formal language $L(x)$}

As we have anticipated in Section 1, we intend to introduce in the present paper a general probabilistic framework that may characterize a class of theories including QM. Of course, this will be done by bearing in mind all the suggestions following from our remarks on QM in Section 2.

As a first step we construct in this section a formal language $L(x)$ (which formalizes, in the case of QM, an elementary sublanguage of the language of $\mathrm{QM}$, and can be considered a part of the formalism $F$ ). To this end we agree to use standard symbols in set theory and logic. In particular, ${ }^{c}, \cap, \cup, \subset, \backslash, \emptyset$ and $\mathbb{P}(\Psi)$ will denote complementation, intersection, union, inclusion, difference, empty set and power set of the set $\Psi$, respectively. Moreover, $\mathrm{N}$ will denote the set of natural numbers.

Definition 3.1. Let $\mathcal{E}, \mathcal{S}$ and $\mathcal{C}$ be disjoint sets whose elements we call properties, states and $\mu$-contexts, respectively, and let us set

$$
\mathcal{E}_{\mathcal{C}}=\left\{E_{C}=(E, C) \mid E \in \mathcal{E}, C \in \mathcal{C}\right\} .
$$

Then, we denote by $L(x)$ a classical predicate logic, constructed as follows.

Syntax.

(i) An individual variable $x$.

(ii) A set $\Pi=\mathcal{E}_{C} \cup \mathcal{S}$ of monadic predicates.

(iii) Connectives $\neg$ (not), $\wedge$ (and), $\vee$ (or).

(iv) Parentheses (,).

(v) A set $\Psi(x)$ of well formed formulas (wffs), obtained by applying recursively standard formation rules in classical logic (to be precise, for every $A \in \Pi, A(x) \in \Psi(x)$; for every $\alpha(x) \in \Psi(x), \neg \alpha(x) \in \Psi(x)$; for every $\alpha(x), \beta(x) \in \Psi(x), \alpha(x) \wedge \beta(x) \in \Psi(x)$ and $\alpha(x) \vee \beta(x) \in \Psi(x))$

Semantics.

(i) A universe $U$, whose elements we call individual objects.

(ii) An injective mapping

$$
\text { ext }: A \in \Pi \longrightarrow \operatorname{ext}(A) \in \mathbb{P}(U) .
$$


(iii) The boolean sublattice $\Theta=<\operatorname{ext}(\Pi)>=\left(\operatorname{ext}(\Pi),{ }^{c}, \cap, \cup\right)$ of $\mathbb{P}(U)$ generated by $\operatorname{ext}(\Pi)$.

(iv) The surjective mapping (still called ext by abuse of language)

$$
\text { ext }: \alpha(x) \in \Psi(x) \longrightarrow \operatorname{ext}(\alpha(x)) \in \Theta
$$

recursively defined by the following rules:

for every $A \in \Pi, \operatorname{ext}(A(x))=\operatorname{ext}(A)$;

for every $\alpha(x) \in \Psi(x), \operatorname{ext}(\neg \alpha(x))=U \backslash \operatorname{ext}(\alpha(x))=(\operatorname{ext}(\alpha(x)))^{c}$;

for every $\alpha(x), \beta(x) \in \Psi(x)$, ext $(\alpha(x) \wedge \beta(x))=\operatorname{ext}(\alpha(x)) \cap \operatorname{ext}(\beta(x))$ and $\operatorname{ext}(\alpha(x) \vee \beta(x))=\operatorname{ext}(\alpha(x)) \cup \operatorname{ext}(\beta(x))$.

(v) A set $\Sigma$ of interpretations of the variable $x$ such that, for every $\sigma \in \Sigma$,

$$
\sigma: x \in\{x\} \longrightarrow \sigma(x) \in U .
$$

(vi) For every $\sigma \in \Sigma$, a truth assignment

$$
\nu_{\sigma}: \alpha(x) \in \Psi(x) \longrightarrow \nu_{\sigma}(\alpha(x)) \in\{t, f\}
$$

(where $t$ stands for true and $f$ for false), such that $\nu_{\sigma}(\alpha(x))=t$ iff $\sigma(x) \in$ $\operatorname{ext}(\alpha(x))$ (hence $\nu_{\sigma}(\alpha(x))=f$ iff $\left.\sigma(x) \in(\operatorname{ext}(\alpha(x)))^{c}\right)$.

The logical preorder and the Lindenbaum-Tarski algebra of $L(x)$ can then be introduced in a standard way, as follows.

Definition 3.2. We denote by $<$ and $\equiv$ the (reflexive and transitive) relation of logical preorder and the relation of logical equivalence on $\Psi(x)$, respectively, defined by standard rules in classical logic (to be precise, for every $\alpha(x), \beta(x) \in$ $\Psi(x), \alpha(x)<\beta(x)$ iff, for every $\sigma \in \Sigma, \nu_{\sigma}(\beta(x))=t$ whenever $\nu_{\sigma}(\alpha(x))=$ $t$, and $\alpha(x) \equiv \beta(x)$ iff $\alpha(x)<\beta(x)$ and $\beta(x)<\alpha(x)$ ). Moreover we put $\Psi^{\prime}(x)=\Psi(x) / \equiv$ and denote by $<^{\prime}$ the partial order canonically induced by $<$ on $\Psi^{\prime}(x)$. Then $\left(\Psi^{\prime}(x),<^{\prime}\right)$ is a boolean lattice (the Lindenbaum-Tarski algebra of $L(x)$ ) whose operations $\neg^{\prime}, \wedge^{\prime}, \vee^{\prime}$ are canonically induced on $\Psi^{\prime}(x)$ by $\neg, \wedge$, $\checkmark$, respectively).

As stated in Definition 3.1, the language $L(x)$ is a classical predicate logic. It has, however, some innovative features from the point of view of the interpretation $I$. Indeed the words "states", "properties", " $\mu$-contexts" and "individual objects" occur in Definition 3.1 just as nouns of elements of sets, but obviously refer to an interpretation that makes these elements correspond to empirical notions denoted by the same nouns. Then, each state $S$ is classified in $L(x)$ as a predicate, and an elementary wff of the form $S(x)$ (interpreted as "the individual object $x$ is in the state $S$ ") is argument of truth assignments, at variance with widespread views that consider states as possible worlds of a Kripkean semantics in QL (see, e.g., Dalla Chiara et al., 2004). Furthermore properties are not classified as predicates of $L(x)$. Rather, a predicate either is a state or it is a pair $E_{C}=(E, C)$ (an elementary wff of the form $E_{C}(x)$ is then interpreted as "the individual object $x$ has the property $E$ in the context $C$ "). 


\section{A contextual probability structure on $L(x)$}

We state now an assumption that is suggested by our introduction of new entities ( $\mu$-contexts) which do not occur explicitly in the formal apparatus of QM.

Axiom P. A mapping $\xi: \operatorname{ext}(\alpha(x)) \in \Theta \longrightarrow \xi(\operatorname{ext}(\alpha(x))) \in[0,1]$ exists such that $\Phi=(U, \Theta, \xi)$ is a classical probability space 4

Based on Axiom $\mathrm{P}$ we can introduce now a probability measure on $L(x)$ by means of the following definition.

Definition 4.1. Let $\Psi^{+}(x) \subset \Psi(x)$ be the set of wffs of $L(x)$ such that, for every $\beta(x) \in \Psi^{+}(x), \xi(\operatorname{ext}(\beta(x)) \neq 0$, and let $p$ be a binary mapping such that

$$
\begin{gathered}
p:(\alpha(x), \beta(x)) \in \Psi(x) \times \Psi^{+}(x) \longrightarrow p(\alpha(x) \mid \beta(x))=\frac{\xi(\operatorname{ext}(\alpha(x)) \cap \operatorname{ext}(\beta(x)))}{\xi(\operatorname{ext}(\beta(x)))} \in \\
{[0,1] .}
\end{gathered}
$$

We say that the pair $(\Phi, p)$ is a $\mu$-contextual probability structure on $L(x)$ and that $p(\alpha(x) \mid \beta(x))$ is the $\mu$-contextual conditional probability of $\alpha(x)$ given $\beta(x)$. Moreover, whenever ext $(\beta(x))=U$ we say that $p(\alpha(x) \mid \beta(x))$ is the $\mu$-contextual absolute probability of $\alpha(x)$ and simply write $p(\alpha(x))$ in place of $p(\alpha(x) \mid \beta(x))$.

The terminology introduced in Definition 4.1 (where the word $\mu$-contextual underlines the dependence of probabilities on $\mu$-contexts through the wffs of $L(x))$, is justified by the following statement.

Proposition 4.1. Let $\beta(x) \in \Psi^{+}(x)$. Then, the mapping

$$
p_{\beta(x)}: \alpha(x) \in \Psi(x) \longrightarrow p(\alpha(x) \mid \beta(x)) \in[0,1]
$$

satisfies the following conditions.

(i) Let $\alpha(x) \in \Psi(x)$ be such that $\operatorname{ext}(\alpha(x))=U$ (equivalently, $\alpha(x) \equiv$ $\alpha(x) \vee \neg \alpha(x))$. Then, $p_{\beta(x)}(\alpha(x))=1$.

(ii) Let $\alpha_{1}(x), \alpha_{2}(x) \in \Psi(x)$ be such that $\operatorname{ext}\left(\alpha_{1}(x)\right) \cap \operatorname{ext}\left(\alpha_{2}(x)\right)=\emptyset$ (equivalently, $\alpha_{1}(x)<\neg \alpha_{2}(x)$ ). Then, $p_{\beta(x)}\left(\alpha_{1}(x) \vee \alpha_{2}(x)\right)=p_{\beta(x)}\left(\alpha_{1}(x)\right)+$ $p_{\beta(x)}\left(\alpha_{2}(x)\right)$.

Proof. Straightforward.

Proposition 4.1 shows indeed that, for every $\beta(x) \in \Psi^{+}(x), p_{\beta(x)}$ is a probability measure on $(\Psi(x), \neg, \wedge, \vee)$.

Examples. Let $E, F \in \mathcal{E}, S, T \in \mathcal{S}, C, D \in \mathcal{C}$, and let $F_{D}(x), S(x) \in \Psi^{+}(x)$. Then, we obtain from Definition 4.1:

(i) $p\left(E_{C}(x) \mid F_{D}(x)\right)=\frac{\xi\left(\operatorname{ext}\left(E_{C}(x)\right) \cap \operatorname{ext}\left(F_{D}(x)\right)\right)}{\xi\left(\operatorname{ext}\left(F_{D}(x)\right)\right)}$;

\footnotetext{
${ }^{4}$ Following a standard terminology we call classical probability space here any triple $(\Omega, \Sigma, \mu)$, where $\Omega$ is a set, $\Sigma$ is a Boolean $\sigma$-subalgebra of $\mathbb{P}(\Omega)$, and $\mu: \Delta \in \Sigma \longrightarrow \mu(\Delta) \in$ $[0,1]$ is a mapping satisfying the following conditions: (i) $\mu(\Omega)=1$; (ii) if $\left\{\Delta_{i}\right\}_{i \in \mathrm{N}}$ is a family of pairwise disjoint elements of $\Sigma$, then $\mu\left(\cup_{i} \Delta_{i}\right)=\Sigma_{i} \mu\left(\Delta_{i}\right)$.
} 
(ii) $p\left(E_{C}(x) \mid S(x)\right)=\frac{\xi\left(\operatorname{ext}\left(E_{C}(x)\right) \cap \operatorname{ext}(S(x))\right)}{\xi(\operatorname{ext}(S(x)))}$;

(iii) $p(T(x) \mid S(x))=\frac{\xi(\operatorname{ext}(T(x)) \cap \operatorname{ext}(S(x)))}{\xi(\operatorname{ext}(S(x)))}$.

Example (iii) is especially interesting because it shows that the $\mu$-contextual conditional probabilities do not always depend on $\mu$-contexts.

By using Axiom $\mathrm{P}$ we have thus introduced $\mu$-contextual conditional and absolute probabilities on $L(x)$. We stress that the $\mu$-contextual probability structure introduced in Definition 4.1 is basically classical, hence these probabilities admit an epistemic interpretation. In other words, they can be considered as indexes of our lack of knowledge of the truth assignments on $L(x)$.

\section{Measurements and mean probabilities}

Based on the notions introduced in Sections 3 and 4, we intend to supply in this section a theoretical description of measurements testing probabilities. To this end, let us observe that our remarks in Section 2 suggest that a test of the probability of a wff $\alpha(x) \in \Psi(x)$ consists in choosing a measurement that checks all the properties that occur in $\alpha(x)$ (hence these properties must be compatible) on an individual object, performing it on a large number of individual objects, and then evaluating the frequencies of the outcomes that have been obtained. Moreover, the theoretical description of this test must refer to a probability measure defined on some set of $\mu$-contexts, to take into account our limited knowledge of the $\mu$-context that must be associated with each implementation of the measurement on an individual object. Bearing in mind these requirements, we introduce the following assumption.

Axiom M. Every $E \in \mathcal{E}$ is associated with a set $\mathcal{M}_{E}$ of measurement procedures 5 and every $M \in \mathcal{M}_{E}$ determines a macroscopic measurement context $C_{M}$ associated with a classical probability space $\left(\mathcal{C}_{M}, \Sigma_{M}, \nu_{M}\right)$, where $\mathcal{C}_{M}$ is a set of $\mu$-contexts and, for every $C \in \mathcal{C}_{M},\{C\}$ belongs to $\Sigma_{M}$.

We have seen in Section 2 that a quantum measurement may require that more than one property be simultaneously tested. We are thus naturally led to introduce the notions of compatibility, testability and conjoint testability in our present framework, as follows.

Definition 5.1. Let $\{E, F, \ldots\}$ be a countable set of properties of $L(x)$. We say that $E, F, \ldots$ are compatible iff $\mathcal{M}_{E} \cap \mathcal{M}_{F} \cap \ldots \neq \emptyset$, and denote by $k$ the compatibility relation on $\mathcal{E}$ defined by setting

$$
\text { for every } E, F \in \mathcal{E}, E k F \text { iff } E \text { and } F \text { are compatible. }
$$

\footnotetext{
${ }^{5}$ We denote here abstract measurement procedures with the same symbols that we have used in Section 2 to denote quantum measurements. We shall see in Section 7 that the latter can be considered as the specific form that the former take in QM.
} 
Moreover, let $\alpha(x) \in \Psi(x)$ and let $E, F, \ldots$ be the properties that occur in the formal expression of $\alpha(x)$ (with indexes in $\mathcal{C}$ ). Then we say that $\alpha(x)$ is testable iff the following conditions hold.

(i) $E, F, \ldots$ are compatible.

(ii) $E, F, \ldots$ occur in the formal expression of $\alpha(x)$ with the same index $C$ and a macroscopic measurement procedure $M \in \mathcal{M}_{E} \cap \mathcal{M}_{F} \cap$... exists such that $C \in \mathcal{C}_{M}$.

Finally, let $\{\alpha(x), \beta(x), \ldots\}$ be a countable set of wffs of $\Psi(x)$, We say that $\alpha(x), \beta(x), \ldots$ are jointly testable iff the wff $\alpha(x) \wedge \beta(x) \wedge \ldots$ is testable. Then we denote by $\Psi^{T}(x)$ the set of all testable propositions of $\Psi(x)$ and, for every $\alpha(x) \in \Psi^{T}(x)$, we write $\alpha_{M}^{C}(x)$ in place of $\alpha(x)$ whenever explicit reference to the measurement procedure $M$ and to the $\mu$-context $C$ defined in (ii) must be done.

We can now state the following proposition.

Proposition 5.1. (i) The binary relation $k$ on $\mathcal{E}$ introduced in Definition 5.1 is reflexive and symmetric, but, generally, not transitive.

(ii) Let $E \in \mathcal{E}, M \in M_{E}$ and $C \in \mathcal{C}_{M}$. Then, $E_{C}(x) \in \Psi^{T}(x)$.

(iii) Let $M$ be a measurement procedure, $C, C^{\prime} \in M$ and $C^{\prime} \neq C$. Moreover, for every $\alpha_{M}^{C}(x) \in \Psi^{T}(x)$, let $\alpha_{M}^{C^{\prime}}(x)$ be the wff obtained from $\alpha_{M}^{C}(x)$ by replacing $C$ with $C^{\prime}$. Then, $\alpha_{M}^{C^{\prime}}(x) \in \Psi^{T}(x)$.

Proof. Straightforward.

Of course, in every theory of the class that we are considering, each measurement procedure $M$ provides a theoretical description, via an empirical interpretation $I$ (see Section 2) of a concrete measurement. Then, it remains to understand what one actually tests when evaluating the frequencies of outcomes obtained as explained above. It is apparent indeed that such a test does not refer to the $\mu$-contextual conditional probabilities introduced in Definition 4.1, because we cannot know nor fix the $\mu$-context associated with each implementation of the measurement (hence $\mu$-contextual probabilities must be classified as theoretical entities that can be interpreted only indirectly, see Section 2). But the unpredictable change of $\mu$-context that generally occurs when performing the measurement on different individual objects suggests that one actually tests a mean of contextual $\mu$-conditional probabilities over the family $\left\{\alpha_{M}^{C}(x)\right\}_{C \in \mathcal{C}_{M}}$. The following definition and assumption formalize this idea.

Definition 5.2. Let $\alpha(x), \beta(x) \in \Psi^{T}(x)$ be jointly testable and let $E, F, \ldots \in \mathcal{E}$ be the properties that occur in one or both the formal expressions of $\alpha(x)$ and $\beta(x)$. Furthermore, let $\beta(x) \in \Psi^{+}(x)$. For every $M \in \mathcal{M}_{E} \cap \mathcal{M}_{F} \cap \ldots$ we put

$$
<p\left(\alpha_{M}^{C}(x) \mid \beta_{M}^{C}(x)\right)>_{\mathcal{C}_{M}}=\sum_{C \in \mathcal{C}_{M}} \nu_{M}(\{C\}) p\left(\alpha_{M}^{C}(x) \mid \beta_{M}^{C}(x)\right) .
$$

Moreover, whenever the following equality holds for every $M, N \in \mathcal{M}_{E} \cap$ $\mathcal{M}_{F} \cap \ldots$

$$
<p\left(\alpha_{M}^{C}(x) \mid \beta_{M}^{C}(x)\right)>_{\mathcal{C}_{M}}=<p\left(\alpha_{N}^{C}(x) \mid \beta_{N}^{C}(x)\right)>_{\mathcal{C}_{N}},
$$


we omit the symbols $M, N, C, D, \mathcal{C}_{M}$ and $\mathcal{C}_{N}$, and say that $\langle p(\alpha(x) \mid \beta(x))>$ is the mean conditional probability of $\alpha(x)$ given $\beta(x)$.

Based on Definition 5.2 we maintain in the following that performing the measurement corresponding (via $I$ ) to a measurement procedure $M \in \mathcal{M}_{E} \cap$ $\mathcal{M}_{F} \cap \ldots$ on a large number of individual objects provides a test of $<p(\alpha(x) \mid$ $\beta(x))>$, or, briefly, a mean probability measurement.

Axiom C. Mean conditional probability (hence mean probability measurements) do exist for every pair $(\alpha(x), \beta(x))$ of jointly testable wffs such that $\beta(x) \in$ $\Psi^{+}(x)$.

It follows from Definition 5.2 and Axiom C that mean conditional probabilities take into account two different kinds of ignorance. First, the lack of knowledge about the truth assignments on $L(x)$ mentioned at the end of Section 4. Second, the ignorance of the $\mu$-context to be associated with a measurement when this measurement is performed. Hence mean conditional probabilities admit an epistemic interpretation even if they are not bound to satisfy Kolmogorov's axioms, for they are average quantities.

To close this section, let us observe that our present perspective is supported by some previous research in the literature. Indeed, as we have anticipated in Section 1, mean conditional probabilities and mean probability measurements are conceptually similar to the universal averages and the universal measurements, respectively, introduced by Aerts and Sassoli de Bianchi (2014, 2017). Moreover, the recognition that two kinds of lack of knowledge occur when a measurement is performed fits in well with similar remarks of these authors 6

\section{Q-probability}

The set $\mathcal{E}$ of all properties has a relevant role in $\mathrm{QM}$, hence we focus on it in the present section.

By using the notion of mean conditional probability introduced in Section 5 , we firstly define an order structure on $\mathcal{E}$, as follows.

Definition 6.1. Let $E \in \mathcal{E}, M \in \mathcal{M}_{E}, C \in \mathcal{C}_{M}, S \in \mathcal{S}$, let $S(x) \in \Psi^{+}(x)$, and let

$$
P_{S}: E \in \mathcal{E} \longrightarrow P_{S}(E) \in[0,1]
$$

be the mapping defined by setting

\footnotetext{
${ }^{6}$ We recall that the Aerts and Sassoli de Bianchi proposal finds its roots in the hidden measurement approach (see, e.g., Aerts, 1986). This approach led the author to introduce state property systems (see, e.g., Aerts, 1999), that successively evolved in the state-contextproperty (SCoP) formalism (see, e.g., Aerts and Gabora, 2005; this formalism was mainly used for working out a theory of concepts, in particular in the field of quantum cognition). It is then possible to show that the SCoP formalism can be (partially) translated into the formalism developed in the present paper, and conversely, which explains the conceptual similarities pointed out above. For the sake of brevity we do not deal with this issue in detail here.
} 


$$
\begin{gathered}
P_{S}(E)=<p\left(E_{C}(x) \mid S(x)\right)>=\sum_{C \in \mathcal{C}_{M}} \nu_{M}(\{C\}) p\left(E_{C}(x) \mid S(x)\right)= \\
\sum_{C \in \mathcal{C}_{M}} \nu_{M}(\{C\}) \frac{\xi\left(E x t\left(E_{C}(x)\right) \cap E x t(S(x))\right)}{\xi(\operatorname{Ext}(S(x)))} .
\end{gathered}
$$

Then, we denote by $\prec$ and $\approx$ the preorder and the equivalence relation on $\mathcal{E}$, respectively, defined by setting, for every $E, F \in \mathcal{E}$,

$$
E \prec F \text { iff, for every } S \in \mathcal{S}, P_{S}(E) \leq P_{S}(F)
$$

and

$$
E \approx F \text { iff } E \prec F \text { and } F \prec E
$$

It is now important to consider a special case that allows us to connect our present framwork with QM. We therefore introduce the following definition.

Definition 6.2. Let $\prec$ be a partial order on $\mathcal{E}$ and let $(\mathcal{E}, \prec)$ be an orthocomplemented lattice. We denote meet, join, orthocomplementation, least element and greatest element of $(\mathcal{E}, \prec)$ by $\cap, \cup,{ }^{\perp}, \mathrm{O}$ and $\mathrm{U}$, respectively. Moreover, we denote by $\perp$ the (binary) orthogonality relation canonically induced by ${ }^{\perp}$ on $(\mathcal{E}, \cap, \uplus, \perp) 7$. Then, for every $S \in \mathcal{S}$, we say that $P_{S}$ is a generalized probability measure on $\left(\mathcal{E}, \cap, \Theta,{ }^{\perp}\right)$ iff it satisfies the following conditions.

(i) $P_{S}(\mathrm{U})=1$.

(ii) If $\left\{E_{1}, E_{2}, \ldots\right\}$ is a countable set of properties of $\mathcal{E}$ and $E_{1}, E_{2}, \ldots$ are pairwise disjoint (i.e., for every $k, l, E_{k} \perp E_{l}$ ), then

$$
P_{S}\left(\uplus_{k} E_{k}\right)=\sum_{k} P_{S}\left(E_{k}\right) .
$$

Whenever $P_{S}$ is a generalized probability measure on $\left(\mathcal{E}\right.$, ก, $\left.\Psi^{\perp},{ }^{\perp}\right)$, for every $E \in \mathcal{E}$ we say that $P_{S}(E)$ is the Q-probability of $E$ given $S$.

Definition 6.2 implies that a generalized probability measure $P_{S}$ does not satisfy Kolmogorov's axioms if $\left(\mathcal{E}, \cap, \Theta,,^{\perp}\right)$ is not a boolean lattice. Nevertheless the Q-probability $P_{S}(E)$ of a property $E \in \mathcal{E}$ given $S$ admits an epistemic interpretation and can be empirically checked, as it is a special case of the mean conditional probability introduced in Definition 5.2. It is then natural to wonder whether a conditional $Q$-probability of a property $E \in \mathcal{E}$ given another property $F \in \mathcal{E}$ can be defined by means of $P_{S}$, generalizing standard procedures in classical propositional logic. But if one tries to put

$$
P_{S}(E \mid F)=\frac{P_{S}(E \cap F)}{P_{S}(F)},
$$

then the mapping

$$
P_{S F}: E \in \mathcal{E} \longrightarrow P_{S}(E \mid F) \in[0,1]
$$

\footnotetext{
${ }^{7}$ We recall that ${ }^{\perp}$ is a unary operation on $(\mathcal{E}, \prec)$ such that, for every $E, F \in \mathcal{E}, E^{\perp \perp}=E$, $E \prec F$ implies $F^{\perp} \prec E^{\perp}, E$ ก $E^{\perp}=O$ and $E$ ש $E^{\perp}=I$. Then $\perp$ is the non-reflexive and symmetric binary relation on $\mathcal{E}$ defined by setting, for every $E, F \in \mathcal{E}, E \perp F$ iff $E \prec F^{\perp}$.
} 
is not a generalized probability measure on $\left(\mathcal{E}\right.$, ก, $\left.\mathbb{U}^{\Perp},{ }^{\perp}\right)$ whenever this lattice is not boolean. Indeed, consider a property $E=E_{1} \uplus E_{2}$, with $E_{1}, E_{2} \in \mathcal{E}$ and $E_{1} \perp E_{2}$. Then, we obtain

$$
P_{S F}(E)=P_{S F}\left(E_{1} \uplus E_{2}\right)=P_{S}\left(E_{1} \uplus E_{2} \mid F\right)=\frac{P_{S}\left(\left(E_{1} \uplus E_{2}\right) \Subset F\right)}{P_{S}(F)},
$$

which is generally different from

$$
\frac{P_{S}\left(\left(E_{1} \cap F\right) \uplus\left(E_{2} \cap F\right)\right)}{P_{S}(F)}=P_{S}\left(E_{1} \mid F\right)+P_{S}\left(E_{2} \mid F\right)=P_{S F}\left(E_{1}\right)+P_{S F}\left(E_{2}\right)
$$

whenever $\left(\mathcal{E}, \cap, \in,^{\perp}\right)$ is not distributive.

To overcome this difficulty one can intuitively refer to a sequence of two measurements and introduce a non-standard kind of conditional probability, as follows.

Definition 6.3. Let $E \in \mathcal{E}$ and let us put $\mathcal{S}_{E}=\left\{S \in \mathcal{S} \mid P_{S}(E) \neq 0\right\}$. We say that a measurement procedure $M \in \mathcal{M}_{E}$ is of first kind iff it is associated with a mapping

$$
t_{E}: S \in \mathcal{S}_{E} \longrightarrow t_{E}(S) \in \mathcal{S}_{E}
$$

such that $P_{t_{E}(S)}(E)=1$. For every $F \in \mathcal{E}$ we then put

$$
P_{S}(F \| E)=P_{t_{E}(S)}(F) .
$$

Moreover, let $(\mathcal{E}, \prec)$ be an orhocomplemented lattice and let $P_{S}$ and $P_{t_{E}(S)}$ be generalized probability measures on $(\mathcal{E}, \prec)$. Then we say that $P_{S}(E \| F)$ is the conditional Q-probability of $E$ given $F$ and $S$.

If a measurement corresponding (via $I$ ) to a first kind measurement procedure $M \in M_{E}$ exists and the conditions at the end of Definition 6.3 are fulfilled, then $P_{S}(E \| F)$ can be tested whenever $S \in \mathcal{S}_{F}$, as Axiom C implies that $P_{t_{E}(S)}(E)$ can always be tested (but no analogous of the Bayes theorem can be stated for conditional Q-probabilities). Definition 6.3 thus introduces a non-standard conditional probability on $(\mathcal{E}, \prec)$ that coexists with the (classical) $\mu$-conditional probability introduced in Definition 4.1 (which instead cannot be tested directly and has the status of a purely theoretical notion, as we have seen in Section 6).

\section{Back to QM}

Axiom $\mathrm{P}$ in Section 4 and axioms $\mathrm{M}$ and $\mathrm{C}$ in Section 5 characterize a broad class $\mathcal{T}$ of theories, even if they have been introduced mainly by bearing in mind QM. They do not occur in the standard formulation of QM, but if we assume that they underlie QM, so that QM belongs to $\mathcal{T}$, we can explain some relevant aspects of $\mathrm{QM}$ in terms of the general notions characterizing $\mathcal{T}$ and obtain a new perspective on quantum probability. 
To attain these results let us firstly recall that in Hibert space QM the following mathematical representation is adopted.

Entity (physical system) $\Longrightarrow$ Hilbert space $\mathcal{H}$.

State $S \in \mathcal{S} \Longrightarrow$ Density operator $\rho_{S}$ on $\mathcal{H}$.

Property $E \in \mathcal{E} \Longrightarrow$ Orthogonal projection operator $P_{E}$ on $\mathcal{H}$.

Furthermore, the set of all orthogonal projection operators on $\mathcal{H}$ is an orthomodular lattice in which the partial order is defined independently of any probability measure. Hence, the representation above induces on $\mathcal{E}$ an order, that we denote by $\ll$, and $(\mathcal{E}, \ll)$ is an orthomodular lattice.

Secondly, let us recall that the Born rule associates a probability value $\operatorname{Tr}\left[\rho_{S} P_{E}\right]$ (that does not depend on any context) with the pair $(E, S)$. Hence a quantum probability

$$
Q_{S}: E \in \mathcal{E} \longrightarrow \operatorname{Tr}\left[\rho_{S} P_{E}\right] \in[0,1]
$$

is defined which is said to be a generalized probability measure on $(\mathcal{E}, \ll)$ (see, e.g., Beltrametti and Cassinelli, 1981). Moreover, the family $\left\{Q_{S}\right\}_{S \in \mathcal{S}}$ is ordering on $(\mathcal{E}, \ll)$ (ibid.), which means that the order induced by it on $\mathcal{E}$ coincides with $\ll$. Therefore the lattice structure of $(\mathcal{E}, \ll)$ can be seen as induced by $\left\{Q_{S}\right\}_{S \in \mathcal{S}}$.

Based on the above remarks, and assuming that QM belongs to $\mathcal{T}$, the order $\ll$ and the quantum probability $Q_{S}$ can be considered as the specific forms that the order $\prec$ and the mapping $P_{S}$ (see Definition 6.1), respectively, take in QM. We thus obtain

$$
P_{S}(E)=P(E \mid S)=Q_{S}(E)=\operatorname{Tr}\left[\rho_{S} P_{E}\right] .
$$

If the quantum probability $Q_{S}$ replaces $P_{S}$ in the conditions (i) and (ii) stated in Definition 6.2, then these conditions are satisfied, which makes the above classification of $Q_{S}$ as a generalized probability measure consistent with Definition 6.2.

The above interpretation of quantum probability leads to consider it as a mean conditional probability (see Definition 5.2). This explains its non-classical character and shows that it can be considered epistemic, at variance with its standard ontic interpretation (see Section 5). Our main goal in this paper has thus been achieved.

Let us denote now by $\kappa$ the compatibility relation introduced in QM on the set of all properties by setting, for every pair $(E, F)$ of properties, $E \kappa F$ iff $\left[P_{E}, P_{F}\right]=0$. This relation is reflexive and symmetric but not transitive. Hence it can be considered as the specific form that the relation $k$ introduced in Definition 5.1 takes in QM.

Coming to quantum measurements, let us remind that first kind quantum measurements exist in QM (see e.g., Piron, 1976; Beltrametti and Cassinelli, 1981), and that the Lüders rule states that, whenever a first kind (ideal) quantum measurement of a property $E$ is performed on an individual object $x$ in the state $S$ and the yes outcome is obtained, then the state of the object after the 
measurement is described by the density operator $\frac{P_{E} \rho_{S} P_{E}}{\operatorname{Tr}\left[\rho_{S} P_{E}\right]}$. Let us therefore denote by $D(\mathcal{H})$ the set of all density operators on $\mathcal{H}$. Then the mapping

$$
\tau_{E}: \rho_{S} \in D(\mathcal{H}) \longrightarrow \tau_{E}\left(\rho_{S}\right)=\frac{P_{E} \rho_{S} P_{E}}{\operatorname{Tr}\left[\rho_{S} P_{E}\right]} \in D(\mathcal{H})
$$

can be considered as the specific form that the mapping $t_{E}$ introduced in Definition 6.3 takes in QM.

Finally, we recall that the conditional probability $Q_{S}(E \mid F)$, in a state $S$, of a property $E$ given a property $F$, is defined in QM by referring to a quantum measurement of $E$ after a quantum measurement of $F$ on an individual object in the state $S$, and it is given by $\frac{\operatorname{Tr}\left[P_{E} P_{F} \rho_{S} P_{F} P_{E}\right]}{\operatorname{Tr}\left[P_{E} \rho_{S} P_{E}\right]}$. Hence this quantity can be considered as the specific form that the conditional Q-probability of $E$ given $F$ and $S$ introduced in Definition 6.3 takes in QM. We thus obtain

$$
P_{S}(E \| F)=Q_{S}(E \mid F)=\frac{\operatorname{Tr}\left[P_{E} P_{F} \rho_{S} P_{F} P_{E}\right]}{\operatorname{Tr}\left[P_{E} \rho_{S} P_{E}\right]}
$$

\section{Closing remarks}

As we have observed in Sections 1 and 5, our mean conditional probabilities and mean probability measurements are conceptually similar to the universal averages and universal measurements, respectively, introduced by Aerts and Sassoli de Bianchi $(2014,2017)$. In particular, our recognition that mean conditional probability summarizes two kinds of lack of knowledge fits in well with the perspective of these authors. However, Aerts and Sassoli de Bianchi uphold that their proposal leads to a possible solution of the quantum measurement problem. Our approach, instead, has been conceived to show that nonclassical (yet epistemic) probabilities may occur as a consequence of contextuality in a broad class of theories. By assuming that QM belongs to this class we obtain an explanation of some typical features of QM in terms of more primitive notions. In particular, the compatibility relation on the set of all physical properties and the quantum notion of conditional probability can be seen as special cases of general notions that can be introduced whenever the links between contextuality and nonclassical probability are recognized. More important, we obtain an epistemic interpretation of quantum probability, notwithstanding its nonclassical structure, that opposes its standard ontic interpretation. We cannot provide instead an explanation of the reduction of the state vector carried out by a quantum measurement in our framework, or avoid the "paradox" of nonlocality of QM (see Section 1 and footnote 3).

Acknowledgement. We thank Dr. Karin Verelst for useful discussions at the Symposium "Worlds of Entanglement" in Brussels. 


\section{BIBLIOGRAPHY}

Aerts, D. (1986). A possible explanation for the probabilities of quantum mechanics. J. Math. Physics 27, 202-210.

Aerts, D. (1999). Foundations of quantum physics: a general realistic and operational approach. Int. J. Theor. Phys. 38, 289-358.

Aerts, D. and Gabora, L. (2005). A state-context-property model of concepts and their combinations ii: A Hilbert space representation. Kibernetes 34, 176204.

Aerts, D. and Sassoli de Bianchi, M. (2014). The extended Bloch representation of quantum mechanics and the hidden-measurement solution of the measurement problem. Ann. Phys. 351, 975-1025.

Aerts, D. and Sassoli de Bianchi, M. (2017). Universal Measurements. How to Free Three Birds in One Move. World Scientific, Singapore.

Bell, J.S. (1964). On the Einstein-Podolski-Rosen Paradox. Physics 1, 195200.

Bell, J.S. (1966). On the Problem of Hidden Variables in Quantum Mechanics. Rev. Mod. Phys. 38, 447-452.

Beltrametti, E. and Cassinelli, G. (1981). The Logic of Quantum Mechanics. Reading (MA), Addison-Wesley.

Birkhoff, G. and von Neumann, J. (1936). The Logic of Quantum Mechanics. Ann. Math. 37, 823-843.

Braithwaite, R.B. (1953). Scientific Explanation. Cambridge University Press, Cambridge.

Busch, P., Lahti, P.J. and Mittelstaedt, P. (1996). The Quantum Theory of Measurement. Springer, Berlin.

Carnap, R. (1966). Philosophical Foundations of Physics. Basic Books Inc., New York.

Dalla Chiara, M. L., Giuntini, R. and Greechie, R. (2004). Reasoning in Quantum Theory. Kluwer, Dordrecht.

Feyerabend, F. (1975). Against Method: Outline of an Anarchist Theory of Knowledge. New Left Books, London.

Garola, C. (1999). Semantic realism: a new philosophy for quantum physics. Int. J. Theor. Phys. 38, 3241-3252.

Garola, C. and Pykacz, J. (2004). Locality and measurement within the SR model for an objective interpretation of quantum mechanics. Found. Phys. 34, 449-475.

Garola, C. and Persano, M. (2014). Embedding quantum mechanics into a broader noncontextual theory. Found. Sci. 19, 217-239.

Garola, C. and Sozzo, S. (2010). Realistic aspects in the standard interpretation of quantum mechanics. Humana.ment. J. Phil. Stud. 13, 81-101.

Garola, C. and Sozzo, S. (2013). Recovering quantum logic within an extended classical framework. Erkenntnis 78, 399-314.

Garola, C., Sozzo, S. and Wu, J. (2016). Outline of a generalization and a reinterpretation of quantum mechanics recovering objectivity. Int. J. Theor. Phys. 55, 2500-2528. 
Greenberger, D.M., Horne, M.A., Shimony, A. and Zeilinger, A. (1990). Bell's theorem without inequalities. Am. J. Phys. 58, 1131-1143.

Hempel, C. C. (1965). Aspects of Scientific Explanation. Free Press, New York.

Kochen, S. and Specker, E. P. (1967). The Problem of Hidden Variables in Quantum Mechanics. J. Math. Mech. 17, 59-87.

Khrennikov, A. (2009). Contextual approach to quantum formalism. Springer, New York.

Kuhn, T.S. (1962). The Structure of Scientific Revolution. Chicago University Press, Chicago.

Ludwig, G. (1983). Foundations of Quantum Mechanics I. Springer, New York.

Mermin, N.D. (1993). Hidden variables and the two theorems of John Bell. Rev. Mod. Phys. 65, 803-815.

Piron, C. (1976). Foundations of Quantum Physics. Benjamin, Reading (MA). 